\title{
On Finite Bases for Weak Semantics: Failures versus Impossible Futures ${ }^{\star}$
}

\author{
Taolue Chen ${ }^{1}$, Wan Fokkink ${ }^{1,2} \&$ Rob van Glabbeek ${ }^{3,4}$ \\ ${ }^{1}$ CWI, Department of Software Engineering, PO Box 94079, 1090 GB Amsterdam, \\ The Netherlands \\ 2 Vrije Universiteit Amsterdam, Department of Theoretical Computer Science, \\ De Boelelaan 1081a, 1081 HV Amsterdam, The Netherlands \\ 3 National ICT Australia, Locked Bag 6016, Sydney, NSW 1466, Australia \\ 4 The University of New South Wales, School of Computer Science and Engineering, \\ Sydney, NSW 2052, Australia
}

\begin{abstract}
We provide a finite basis for the (in)equational theory of the process algebra BCCS modulo the weak failures preorder and equivalence. We also give positive and negative results regarding the axiomatizability of BCCS modulo weak impossible futures semantics.
\end{abstract}

\section{Introduction}

Labeled transition systems constitute a widely used model of concurrent computation. They model processes by explicitly describing their states and their transitions from state to state, together with the actions that produce these transitions. Several notions of behavioral semantics have been proposed, with the aim to identify those states that afford the same observations $[9,11]$. For equational reasoning about processes, one needs to find an axiomatization that is sound and ground-complete modulo the semantics under consideration, meaning that all equivalent closed terms can be equated. Ideally, such an axiomatization is also $\omega$-complete, meaning that all equivalent open terms can be equated. If such a finite axiomatization exists, it is said that there is a finite basis for the equational theory.

For concrete semantics, so in the absence of the silent action $\tau$, the existence of finite bases is well-studied $[5,11,13]$, in the context of the process algebra BCCSP, containing the basic process algebraic operators from CCS and CSP. However, for weak semantics, that take into account the $\tau$, hardly anything is known on finite bases. In [9], Van Glabbeek presented a spectrum of weak semantics. For several of the semantics in this spectrum, a sound and ground-complete axiomatization has been given, in the setting of the process algebra BCCS (BCCSP extended by $\tau$ ), see, e.g., [10]. But only for weak impossible futures semantics has a finite basis been given [17], for BCCS, in case of an infinite alphabet of actions. The reason for this lack of results on finite bases, apart from the inherent difficulties arisen with weak semantics, may be that it is usually not so straightforward to define a notion of unique normal form for open terms in a weak semantics. Here we will employ a saturation technique, in which normal forms

\footnotetext{
* This work is partially supported by the Dutch Bsik project BRICKS.
} 
are saturated with subterms (instead of the usual approach, in which normal forms are made as small as possible).

In this paper, we focus on two closely related weak semantics, based on failures and impossible futures. A weak failure consists of a trace $a_{1} \cdots a_{n}$ and a set $A$, both of concrete actions. A state exhibits this weak failure pair if it can perform the trace $a_{1} \cdots a_{n}$ (possibly intertwined with $\tau$ 's) to a state that cannot perform any action in $A$ (even after performing $\tau$ 's). In a weak impossible future, $A$ can be a set of traces. Weak failures semantics plays an essential role for the process algebra CSP [2]. For convergent processes, it coincides with testing semantics [6,14], and thus is the coarsest congruence for the CCS parallel composition that respects deadlock behavior. Weak impossible futures semantics [16] is a natural variant of possible futures semantics [15]. In [12] it is shown that weak impossible futures semantics, with an additional root condition, is the coarsest congruence containing weak bisimilarity with explicit divergence that respects deadlock/livelock traces (or fair testing, or any liveness property under a global fairness assumption) and assigns unique solutions to recursive equations.

The heart of our paper is a finite basis for the inequational theory of BCCS modulo the weak failures preorder. The axiomatization consists of the standard axioms A1-4 for bisimulation, three extra axioms WF1-3 for failures semantics, and in case of a finite alphabet $A$, an extra axiom $\mathrm{WF}_{A}$. The proof that A1-4 and WF1-3 are a finite basis in case of an infinite alphabet is actually a sub-proof of the proof that A1-4, WF1-3 and $\mathrm{WF}_{A}$ are a finite basis in case of a finite alphabet. Pivotal for this proof is the construction of "saturated" sets of actions within a term; this notion was introduced in [6]. Since here we want to obtain an $\omega$-completeness result, we need to extend this notion to variables. We also apply an algorithm from $[1,8]$ to obtain a finite basis for BCCS modulo weak failures equivalence for free.

At the end, we investigate the equational theory of BCCS modulo weak impossible futures semantics. This shows a remarkable difference with weak failures semantics, in spite of the strong similarity between the definitions of these semantics (and between their ground-complete axiomatizations). As said, in case of an infinite alphabet, BCCS modulo the weak impossible futures preorder has a finite basis [17]. However, we show that in case of a finite alphabet, such a finite basis does not exist. Moreover, in case of weak impossible futures equivalence, there is no ground-complete axiomatization, regardless of the cardinality of the alphabet.

A finite basis for the equational theory of BCCSP modulo (concrete) failures semantics was given in [7]. And the equational theory of BCCSP modulo (concrete) impossible futures semantics is studied in [3]. It is interesting to see that our results for weak semantics coincide with their concrete counterparts, which raises some challenging open question: can one establish a general theorem to link the axiomatizability (or nonaxiomatizability) of concrete and weak semantics? We conjecture that this might be relatively easier for the semantics in the linear-time spectrum while much more difficult for the ones in the branching-time spectrum.

Due to space restriction, some proofs, remarks and examples are omitted in the current paper. These include, in particular, proofs of Lem. 1, Lem. 3 and those in Sec. 4. However, they can be found in the full version of this paper [4]. 


\section{Preliminaries}

$\operatorname{BCCS}(A)$ is a basic process algebra for expressing finite process behavior. Its signature consists of the constant $\mathbf{0}$, the binary operator ${ }_{-}+_{-}$, and unary prefix operators $\tau_{-}$and $a_{-}$, where $a$ is taken from a nonempty set $A$ of visible actions, called the alphabet, ranged over by $a, b, c$. We assume that $\tau \notin A$ and write $A_{\tau}$ for $A \cup\{\tau\}$, ranged over by $\alpha$.

$$
t::=0|a t| \tau t|t+t| x
$$

Closed $\operatorname{BCCS}(A)$ terms, ranged over by $p, q$, represent finite process behaviors, where $\mathbf{0}$ does not exhibit any behavior, $p+q$ offers a choice between the behaviors of $p$ and $q$, and $\alpha p$ executes action $\alpha$ to transform into $p$. This intuition is captured by the transition rules below, in which $\alpha$ ranges over $A_{\tau}$. They give rise to $A_{\tau}$-labeled transitions between closed BCCS terms.

$$
\overline{\alpha x \stackrel{\alpha}{\rightarrow} x} \quad \frac{x \stackrel{\alpha}{\rightarrow} x^{\prime}}{x+y \stackrel{\alpha}{\rightarrow} x^{\prime}} \quad \frac{y \stackrel{\alpha}{\rightarrow} y^{\prime}}{x+y \stackrel{\alpha}{\rightarrow} y^{\prime}}
$$

We assume a countably infinite set $V$ of variables; $w, x, y, z$ denote elements of $V$. Open BCCS terms, denoted by $t, u, v$, may contain variables from $V$. It is technically convenient to extend the operational semantics to open terms. We do not include additional rules for variables. We write $t \Rightarrow u$ if there is a sequence of $\tau$-transitions $t \stackrel{\tau}{\rightarrow} \cdots \stackrel{\tau}{\rightarrow} u$.

The depth of a term $t$, denoted by $|t|$, is the length of the longest trace of $t$, not counting $\tau$-transitions. It is defined inductively as follows: $|\mathbf{0}|=|x|=0 ;|a t|=1+|t|$; $|\tau t|=|t| ;|t+u|=\max \{|t|,|u|\}$.

A (closed) substitution, denoted by $\rho, \sigma$, maps variables in $V$ to (closed) terms. For open terms $t$ and $u$, and a preorder $\preceq$ (or equivalence $\simeq$ ) on closed terms, we define $t \preceq u$ (or $t \simeq u$ ) if $\rho(t) \preceq \rho(u)$ (resp. $\rho(t) \simeq \rho(u)$ ) for all closed substitutions $\rho$. Clearly, $t \stackrel{a}{\rightarrow} t^{\prime}$ implies that $\sigma(t) \stackrel{a}{\rightarrow} \sigma\left(t^{\prime}\right)$ for all substitutions $\sigma$.

An axiomatization is a collection of equations $t \approx u$ or of inequations $t \preccurlyeq u$. The (in)equations in an axiomatization $E$ are referred to as axioms. If $E$ is an equational axiomatization, we write $E \vdash t \approx u$ if the equation $t \approx u$ is derivable from the axioms in $E$ using the rules of equational logic (reflexivity, symmetry, transitivity, substitution, and closure under BCCS contexts). For the derivation of an inequation $t \preccurlyeq u$ from an inequational axiomatization $E$, denoted by $E \vdash t \preccurlyeq u$, the rule for symmetry is omitted. We will also allow equations $t \approx u$ in inequational axiomatizations, as an abbreviation of $t \preccurlyeq u$ and $u \preccurlyeq t$.

An axiomatization $E$ is sound modulo a preorder $\preceq$ (or equivalence $\simeq$ ) if for any terms $t, u$, from $E \vdash t \preccurlyeq u$ (or $E \vdash t \approx u$ ) it follows that $t \preceq u$ (or $t \simeq u$ ). $E$ is groundcomplete for $\preceq$ (or $\simeq$ ) if for any closed terms $p, q, p \preceq q$ (or $p \simeq q$ ) implies $E \vdash p \preccurlyeq q$ (or $E \vdash p \approx q$ ). And $E$ is $\omega$-complete if for any terms $t$, $u$ with $E \vdash \rho(t) \preccurlyeq \rho(u)$ (or $E \vdash \rho(t) \approx \rho(u)$ ) for all closed substitutions $\rho$, we have $E \vdash t \preccurlyeq u$ (or $E \vdash t \approx u$ ). The equational theory of BCCS modulo a preorder $\preceq$ (or equivalence $\simeq$ ) is said to be finitely based if there exists a finite, $\omega$-complete axiomatization that is sound and ground-complete for BCCS modulo $\preceq$ (or $\simeq$ ). 
A1-4 below are the core axioms for BCCS modulo bisimulation semantics. We write $t=u$ if $\mathrm{A} 1-4 \vdash t \approx u$.

$$
\begin{aligned}
& \text { A1 } x+y \approx y+x \\
& \text { A2 }(x+y)+z \approx x+(y+z) \\
& \text { A3 } x+x \approx x \\
& \mathrm{~A} 4 \quad x+\mathbf{0} \approx x
\end{aligned}
$$

Summation $\sum_{i \in\{1, \ldots, n\}} t_{i}$ denotes $t_{1}+\cdots+t_{n}$, where summation over the empty set denotes $\mathbf{0}$. As binding convention, _ $+_{-}$and summation bind weaker than $\alpha_{-}$. For every term $t$ there exists a finite set $\left\{\alpha_{i} t_{i} \mid i \in I\right\}$ of terms and a finite set $Y$ of variables such that $t=\sum_{i \in I} \alpha_{i} t_{i}+\sum_{y \in Y} y$. The $\alpha_{i} t_{i}$ for $i \in I$ and the $y \in Y$ are called the summands of $t$. When $Y$ is a set of variables, we often denote the term $\sum_{y \in Y} y$ by $Y$.

Definition 1 (Initial actions). For any term $t$, the set $\mathcal{I}(t)$ of initial actions is defined as $\mathcal{I}(t)=\{a \in A \mid t \Rightarrow \stackrel{a}{\rightarrow}\}$.

\section{Definition 2 (Weak failures).}

- A pair $\left(a_{1} \cdots a_{k}, B\right)$, with $k \geq 0$ and $B \subseteq A$, is a weak failure pair of a process $p_{0}$ if there is a path $p_{0} \Rightarrow \stackrel{a_{1}}{\rightarrow} \Rightarrow \cdots \Rightarrow \stackrel{a_{k}}{\rightarrow} \Rightarrow p_{k}$ with $\mathcal{I}\left(p_{k}\right) \cap B=\emptyset$.

- $p \precsim$ WF $q$ if the weak failure pairs of $p$ are also weak failure pairs of $q$.

- $p \preceq_{\mathrm{WF}} q$ if (1) $p \precsim_{\mathrm{WF}} q$ and (2) $p \stackrel{\tau}{\rightarrow}$ implies that $q \stackrel{\tau}{\rightarrow}$.

$-\simeq_{\mathrm{WF}}=\preceq_{\mathrm{WF}} \cap \preceq_{\mathrm{WF}}^{-1}$.

$\preceq_{\mathrm{WF}}$ is a precongruence for BCCS, meaning that $p_{1} \preceq_{\mathrm{WF}} q_{1}$ and $p_{2} \preceq_{\mathrm{WF}} q_{2}$ implies $p_{1}+p_{2} \preceq_{\mathrm{WF}} q_{1}+q_{2}$ and $\alpha p_{1} \preceq_{\mathrm{WF}} \alpha q_{1}$ for $\alpha \in A_{\tau}$. Likewise, $\simeq_{\mathrm{WF}}$ is a congruence for BCCS.

\section{A Finite Basis for Weak Failures Semantics}

\subsection{Axioms for the Weak Failures Preorder}

$$
\begin{array}{rrrl}
\text { WF1 } & a x+a y & \approx a(\tau x+\tau y) \\
\text { WF2 } & \tau(x+y) & \preccurlyeq \tau x+y \\
\text { WF3 } & x & \preccurlyeq \tau x+y
\end{array}
$$

Table 1. Axiomatization for the weak failures preorder

An axiomatization for $\preceq_{\mathrm{WF}}$ is presented in Tab. 1. It is not hard to see that A1-4+WF1-3 is sound and ground-complete for $\operatorname{BCCS}(A)$ modulo $\preceq_{\mathrm{WF}}$ (cf. [6]).

Theorem 1. A1-4+WF1-3 is sound and ground-complete for BCCS $(A)$ modulo $\preceq \mathrm{WF}$.

In this section, we extend this completeness result with two $\omega$-completeness results. The first one says, in combination with Theorem 1, that as long as our alphabet of actions is infinite, the axioms A1-4+WF1-3 constitute a finite basis for the inequational theory of $\operatorname{BCCS}(A)$ modulo $\preceq$ WF. 
Theorem 2. If $|A|=\infty$, then A1-4+WF1-3 is $\omega$-complete for BCCS(A) modulo $\preceq \mathrm{WF}$.

To get a finite basis for the inequational theory of BCCS modulo $\preceq_{\mathrm{WF}}$ in case $|A|<\infty$, we need to add the following axiom:

$$
\mathrm{WF}_{A} \quad \sum_{a \in A} a x_{a} \preccurlyeq \sum_{a \in A} a x_{a}+y
$$

where the $x_{a}$ for $a \in A$ and $y$ are distinct variables.

Theorem 3. If $|A|<\infty$, then $A 1-4+W F 1-3+W F_{A}$ is $\omega$-complete for BCCS( $A$ ) modulo $\preceq$ WF.

For a start, the inequations in Tab. 2 can be derived from A1-4+WF1-3:

$$
\begin{aligned}
& \text { D1 } \tau(x+y)+x \approx \tau(x+y) \\
& \text { D2 } \quad \tau(\tau x+y) \approx \tau x+y \\
& \text { D3 } a x+\tau(a y+z) \approx \tau(a x+a y+z) \\
& \text { D4 } \tau x \preccurlyeq \tau x+y \\
& \text { D5 } \quad \sum_{i \in I} a x_{i} \approx a\left(\sum_{i \in I} \tau x_{i}\right) \text { for finite index sets } I \\
& \text { D6 } \quad \tau x+y \approx \tau x+\tau(x+y)
\end{aligned}
$$

Table 2. Derived inequations

Lemma 1. D1-D6 are derivable from A1-4+WF1-3.

Proof. Cf. [4].

\subsection{Normal Forms}

The notion of a normal form, which is formulated in the following two definitions, will play a key role in the forthcoming proofs. For any set $L \subseteq A \cup V$ of actions and variables let $A_{L}=L \cap A$, the set of actions in $L$, and $V_{L}=L \cap V$, the set of variables in $L$.

Definition 3 (Saturated family). Suppose $\mathcal{L}$ is a finite family of finite sets of actions and variables. We say $\mathcal{L}$ is saturated if it is nonempty and

- $L_{1}, L_{2} \in \mathcal{L}$ implies that $L_{1} \cup L_{2} \in \mathcal{L}$; and

- $L_{1}, L_{2} \in \mathcal{L}$ and $L_{1} \subseteq L_{3} \subseteq L_{2}$ imply that $L_{3} \in \mathcal{L}$.

\section{Definition 4 (Normal form).}

(i) A term $t$ is in $\tau$ normal form if

$$
t=\sum_{L \in \mathcal{L}} \tau\left(\sum_{a \in A_{L}} a t_{a}+V_{L}\right)
$$

where the $t_{a}$ are in normal form and $\mathcal{L}$ is a saturated family of sets of actions and variables. We write $L(t)$ for $\bigcup_{L \in \mathcal{L}} L$; note that $L(t) \in \mathcal{L}$. 
(ii) $t$ is in action normal form if

$$
t=\sum_{a \in A_{L}} a t_{a}+V_{L}
$$

where the $t_{a}$ are in normal form and $L \subseteq A \cup V$. We write $L(t)$ for $L$.

(iii) $t$ is in normal form if it is either in $\tau$ normal form or in action normal form.

Remark 1. In the notion of a normal form, it is required that for any $a \in A$, if $t \Rightarrow \stackrel{a}{\rightarrow} t_{1}$ and $t \Rightarrow \stackrel{a}{\rightarrow} t_{2}$, then $t_{1}$ and $t_{2}$ are syntactically identical. Because of this, we can use a more suggestive notation $t_{a}$ to denote the unique term such that $t \Rightarrow \stackrel{a}{\rightarrow} t_{a}$.

We prove that every term can be equated to a normal form.

Lemma 2. For any term $t, \vdash t \approx t^{\prime}$ for some normal form $t^{\prime}$.

Proof. By induction on $|t|$. We distinguish two cases.

$-t \stackrel{\tau}{\nrightarrow}$. Let $t=\sum_{i \in I} a_{i} t_{i}+Y$. By D5,

$$
\vdash t \approx \sum_{a \in \mathcal{I}(t)} a\left(\sum_{i \in I, a_{i}=a} \tau t_{i}\right)+Y
$$

By induction, for each $a \in \mathcal{I}(t)$,

$$
\vdash \sum_{i \in I, a_{i}=a} \tau t_{i} \approx t_{a}
$$

for some normal form $t_{a}$. So we are done.

- $t \stackrel{\tau}{\rightarrow}$. By applying D2, we can derive

$$
\vdash t \approx \sum_{i \in I} \tau t_{i}+\sum_{j \in J} a_{j} t_{j}+X
$$

with $I \neq \emptyset$, where for each $i \in I, t_{i} \stackrel{\tau}{f}$, and thus

$$
t_{i}=\sum_{k \in K_{i}} c_{k} t_{k}^{\prime}+Y_{i}
$$

By (1), (2) and D1,

$$
\vdash t \approx \sum_{i \in I} \tau t_{i}+\sum_{i \in I} \sum_{k \in K_{i}} c_{k} t_{k}^{\prime}+\sum_{j \in J} a_{j} t_{j}+Y
$$

where $Y=X \cup \bigcup_{i \in I} Y_{i}$. For each $a \in \mathcal{I}(t)$, we define

$$
u_{a}=\sum_{i \in I} \sum_{k \in K_{i}, c_{k}=a} c_{k} t_{k}^{\prime}+\sum_{j \in J, a_{j}=a} a_{j} t_{j}
$$


By (3),

$$
\vdash t \approx \sum_{i \in I} \tau t_{i}+\sum_{a \in \mathcal{I}(t)} u_{a}+Y
$$

Define

$$
\mathcal{L}=\left\{L \mid \mathcal{I}\left(t_{i}\right) \cup Y_{i} \subseteq L \subseteq \mathcal{I}(t) \cup Y \text { for some } i \in I\right\}
$$

Clearly, $\mathcal{L}$ is a saturated family. For each $i \in I$, by D3,

$$
\vdash \tau t_{i}+\sum_{a \in \mathcal{I}\left(t_{i}\right)} u_{a} \approx \tau\left(t_{i}+\sum_{a \in \mathcal{I}\left(t_{i}\right)} u_{a}\right)=\tau\left(\sum_{a \in \mathcal{I}\left(t_{i}\right)} u_{a}+Y_{i}\right)
$$

It follows from (5) and (6) that

$$
\vdash t \approx \sum_{i \in I} \tau\left(\sum_{a \in \mathcal{I}\left(t_{i}\right)} u_{a}+Y_{i}\right)+\sum_{a \in \mathcal{I}(t)} u_{a}+Y
$$

For each $L \in \mathcal{L}$, by definition, there exists some $i(L) \in I$ such that $\mathcal{I}\left(t_{i(L)}\right) \cup$ $Y_{i(L)} \subseteq L$. Hence by D6,

$$
\vdash \tau\left(\sum_{a \in \mathcal{I}\left(t_{i(L)}\right)} u_{a}+Y_{i(L)}\right)+\sum_{a \in A_{L}} u_{a}+V_{L} \approx \tau\left(\sum_{a \in \mathcal{I}\left(t_{i(L)}\right)} u_{a}+Y_{i(L)}\right)+\tau\left(\sum_{a \in A_{L}} u_{a}+V_{L}\right)
$$

We note that, since $\mathcal{I}\left(t_{i}\right) \cup Y_{i} \in \mathcal{L}$ for each $i \in I$, not only the second but also the first summand at the right-hand side of (8) is a summand of $\sum_{L \in \mathcal{L}} \tau\left(\sum_{a \in A_{L}} u_{a}+\right.$ $V_{L}$ ). Hence, (using $I \neq \emptyset$ ) it follows from (7) and (8) that

$$
\vdash t \approx \sum_{L \in \mathcal{L}} \tau\left(\sum_{a \in A_{L}} u_{a}+V_{L}\right)
$$

For $a \in \mathcal{I}(t)$, by (4) and D5,

$$
\vdash u_{a} \approx a\left(\sum_{i \in I} \sum_{k \in K_{i}, c_{k}=a} \tau t_{k}^{\prime}+\sum_{j \in J, a_{j}=a} \tau t_{j}\right)
$$

And by induction,

$$
\vdash \sum_{i \in I} \sum_{k \in K_{i}, c_{k}=a} \tau t_{k}^{\prime}+\sum_{j \in J, a_{j}=a} \tau t_{j} \approx t_{a}
$$

for some normal form $t_{a}$. Hence, by (9), (10) and (11),

$$
\vdash t \approx \sum_{L \in \mathcal{L}} \tau\left(\sum_{a \in A_{L}} a t_{a}+V_{L}\right)
$$

This completes the proof.

Lemma 3. Suppose $t$ and $u$ are both in normal forms and $t \preceq_{\mathrm{WF}} u$. If $t \stackrel{a}{\rightarrow} t_{a}$, then there exists a term $u_{a}$ such that $u \Rightarrow \stackrel{a}{\rightarrow} u_{a}$ and $t_{a} \precsim{ }_{\mathrm{WF}} u_{a}$.

Proof. Cf. [4]. 


\section{$3.3 \omega$-Completeness Proof}

We are now in a position to prove Theo. 2 ( $\omega$-completeness in case of an infinite alphabet) and Theo. 3 ( $\omega$-completeness in case of a finite alphabet), along with Theo. 1 (ground completeness). We will prove these three theorems in one go. Namely, in the proof, two cases are distinguished; only in the second case $(\mathcal{I}(t)=A)$, in which the $A$ is guaranteed to be finite, will the axiom $\mathrm{WF}_{A}$ play a role.

Proof. Let $t \preceq_{\mathrm{WF}} u$. We need to show that $\vdash t \preccurlyeq u$. We apply induction on $|t|+|u|$. By Lem. 2, we can write $t$ and $u$ in normal form.

We first prove that $L(t) \subseteq L(u)$. Suppose this is not the case. Then there exists some $a \in A_{L(t)} \backslash A_{L(u)}$ or some $x \in V_{L(t)} \backslash V_{L(u)}$. In the first case, let $\rho$ be the closed substitution with $\rho(w)=\mathbf{0}$ for all $w \in V$; we find that $(a, \emptyset)$ is a weak failure pair of $\rho(t)$ but not of $\rho(u)$, which contradicts the fact that $\rho(t) \preceq$ WF $\rho(u)$. In the second case, pick some $d>\max \{|t|,|u|\}$, and consider the closed substitution $\rho(x)=a^{d} \mathbf{0}$ and $\rho(w)=\mathbf{0}$ for $w \neq x$. Then $\left(a^{d}, \emptyset\right)$ is weak failure pair of $\rho(t)$. However, it can not be a weak failure pair of $\rho(u)$, again contradicting $\rho(t) \preceq$ WF $\rho(u)$.

We distinguish two cases, depending on whether $\mathcal{I}(t)=A$ or not.

1. $\mathcal{I}(t) \neq A$. We distinguish three cases. Due to the condition that $t \stackrel{\tau}{\rightarrow}$ implies $u \stackrel{\tau}{\rightarrow}$, it cannot be the case that $t$ is an action normal form and $u$ a $\tau$ normal form.

(a) $t$ and $u$ are both action normal forms. So $t=\sum_{a \in A_{L}} a t_{a}+V_{L}$ and $u=$ $\sum_{a \in A_{M}} a u_{a}+V_{M}$. We show that $L(t)=L(u)$. Namely, pick $b \in A \backslash A_{L}$, and let $\rho$ be the closed substitution with $\rho(w)=\mathbf{0}$ for any $w \in V_{L}$, and $\rho(w)=b \mathbf{0}$ for $w \notin V_{L}$. As $(\varepsilon, A \backslash \mathcal{I}(t))$ is a weak failure pair of $t$, and hence of $u$, it must be that $L(u) \subseteq L(t)$. Together with $L(t) \subseteq L(u)$ this gives $L(t)=L(u)$. By Lem. 3, for each $a \in \mathcal{I}(t), t_{a} \precsim u_{a}$, and thus clearly $t_{a} \preceq_{\mathrm{WF}} \tau u_{a}$. By induction, $\vdash t_{a} \preccurlyeq \tau u_{a}$ and hence $\vdash a t_{a} \preccurlyeq a u_{a}$. It follows that

$$
\vdash t=\sum_{a \in A_{L}} a t_{a}+V_{L} \preccurlyeq \sum_{a \in A_{L}} a u_{a}+V_{L}=\sum_{a \in A_{M}} a u_{a}+V_{M}=u
$$

(b) Both $t$ and $u$ are $\tau$ normal forms:

$$
t=\sum_{L \in \mathcal{L}} \tau\left(\sum_{a \in A_{L}} a t_{a}+V_{L}\right)
$$

and

$$
u=\sum_{M \in \mathcal{M}} \tau\left(\sum_{a \in A_{M}} a u_{a}+V_{M}\right)
$$

By Lem. 3, for each $a \in \mathcal{I}(t), t_{a} \precsim u_{a}$, and thus clearly $t_{a} \preceq_{\mathrm{WF}} \tau u_{a}$. By induction, $\vdash t_{a} \preccurlyeq \tau u_{a}$. By these inequalities, together with D4,

$$
\vdash t \preccurlyeq \sum_{L \in \mathcal{L}} \tau\left(\sum_{a \in A_{L}} a u_{a}+V_{L}\right)+u
$$

We now show that $\mathcal{L} \subseteq \mathcal{M}$. Take any $L \in \mathcal{L}$, pick $b \in A \backslash A_{L}$, and consider the closed substitution $\rho(w)=\mathbf{0}$ for any $w \in V_{L}$, and $\rho(w)=b \mathbf{0}$ for $w \notin V_{L}$. 
Since $\rho(t) \stackrel{\tau}{\rightarrow} \rho\left(\sum_{a \in L} a t_{a}\right)$ and $\rho(t) \preceq$ WF $\rho(u)$, there exists an $M \in \mathcal{M}$ with $A_{M} \subseteq A_{L}$ and $V_{M} \subseteq V_{L}$. Since also $L \subseteq L(t) \subseteq L(u)$, and $\mathcal{M}$ is saturated, it follows that $L \in \mathcal{M}$. Hence, $\mathcal{L} \subseteq \mathcal{M}$.

Since $\mathcal{L} \subseteq \mathcal{M}$,

$$
\sum_{L \in \mathcal{L}} \tau\left(\sum_{a \in A_{L}} a u_{a}+V_{L}\right)+u=u
$$

By (12) and (13), $\vdash t \preccurlyeq u$.

(c) $t$ is an action normal form and $u$ is a $\tau$ normal form. Then $\tau t \preceq_{\mathrm{WF}} u$. Note that $\tau t$ is a $\tau$ normal form, so according to the previous case,

$$
\vdash \tau t \preccurlyeq u
$$

By WF3,

$$
\vdash t \preccurlyeq \tau t \preccurlyeq u
$$

2. $\mathcal{I}(t)=A$. Note that in this case, $|A|<\infty$. So, according to Theorem 3 , axiom $\mathrm{WF}_{A}$ is at our disposal. As before, we distinguish three cases.

(a) Both $t$ and $u$ are action normal forms. Since $L(t) \subseteq L(u)$ we have $t=$ $\sum_{a \in A} a t_{a}+W$ and $u=\sum_{a \in A} a u_{a}+X$ with $W \subseteq X$. By $\mathrm{WF}_{A}$,

$$
\vdash \sum_{a \in A} a t_{a} \preccurlyeq \sum_{a \in A} a t_{a}+u
$$

By Lem. 3, for each $a \in A, t_{a} \precsim \mathrm{WF} u_{a}$, and thus clearly $t_{a} \preceq_{\mathrm{WF}} \tau u_{a}$. By induction, $\vdash t_{a} \preccurlyeq \tau u_{a}$. It follows, using $W \subseteq X$, that

$$
\vdash t=\sum_{a \in A} a t_{a}+W \preccurlyeq \sum_{a \in A} a u_{a}+u+W=u
$$

(b) Both $t$ and $u$ are $\tau$ normal forms.

$$
t=\sum_{L \in \mathcal{L}} \tau\left(\sum_{a \in A_{L}} a t_{a}+V_{L}\right)
$$

and

$$
u=\sum_{M \in \mathcal{M}} \tau\left(\sum_{a \in A_{M}} a u_{a}+V_{M}\right)
$$

By D1 and $\mathrm{WF}_{A}$ (clearly, in this case $A_{L(t)}=A$ ),

$$
\vdash t \approx t+\sum_{a \in A} a t_{a} \preccurlyeq t+\sum_{a \in A} a t_{a}+u
$$

By Lem. 3, for each $a \in A, t_{a} \precsim \mathrm{WF} u_{a}$, and thus clearly $t_{a} \preceq_{\mathrm{WF}} \tau u_{a}$. By induction, $\vdash t_{a} \preccurlyeq \tau u_{a}$. By these inequalities, together with (14),

$$
\vdash t \preccurlyeq \sum_{L \in \mathcal{L}} \tau\left(\sum_{a \in A_{L}} a u_{a}+V_{L}\right)+\sum_{a \in A} a u_{a}+u
$$


So by D1,

$$
\vdash t \preccurlyeq \sum_{L \in \mathcal{L}} \tau\left(\sum_{a \in A_{L}} a u_{a}+V_{L}\right)+u
$$

Now for $L \in \mathcal{L}$ with $A_{L} \neq A$ we have $L \in \mathcal{M}$ using the same reasoning as in 1(b). For $L \in \mathcal{L}$ with $A_{L}=A$ we have $V_{L} \subseteq V_{L(t)} \subseteq V_{L(u)}$. By $\mathrm{WF}_{A}$ we have

$$
\vdash \tau\left(\sum_{a \in A_{L}} a u_{a}+V_{L}\right) \preccurlyeq \tau\left(\sum_{a \in A} a u_{a}+V_{L(u)}\right)
$$

As the latter is a summand of $u$ we obtain $t \preccurlyeq u$.

(c) $t$ is an action normal form and $u$ is a $\tau$ normal form. This can be dealt with as in case $1(\mathrm{c})$.

This completes the proof.

\subsection{Weak Failures Equivalence}

In $[1,8]$ an algorithm is presented which takes as input a sound and ground-complete inequational axiomatization $E$ for BCCSP modulo a preorder in the linear time - branching time spectrum that includes the ready simulation preorder, and generates as output an equational axiomatization $\mathcal{A}(E)$ which is sound and ground-complete for BCCSP modulo the corresponding equivalence. Moreover, if the original axiomatization $E$ is $\omega$-complete, so is the resulting axiomatization. The axiomatization $\mathcal{A}(E)$ generated by the algorithm from $E$ contains the axioms A1-4 for bisimulation equivalence and the axioms $\beta(\alpha x+z)+\beta(\alpha x+\alpha y+z) \approx \beta(\alpha x+\alpha y+z)$ for $\alpha, \beta \in A_{\tau}$ that are valid in ready simulation semantics, together with the following equations, for each inequational axiom $t \preccurlyeq u$ in $E$ :

- $t+u \approx u$; and

- $\alpha(t+x)+\alpha(u+x) \approx \alpha(u+x)$ (for each $\alpha \in A_{\tau}$, and some variable $x$ that does not occur in $t+u)$.

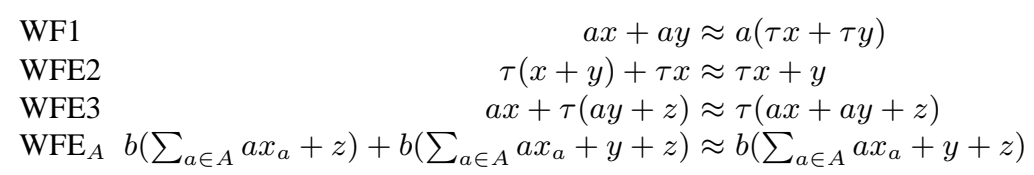

Table 3. Axiomatization for weak failures equivalence

Since weak failures is coarser than (strong) ready simulation, we can run the algorithm from $[1,8]$, and thus, after simplification and omission of redundant axioms, obtain the axiomatization for weak failures equivalence in Tab. 3. The axioms WF1, WFE2-3 already appeared in [10]. A1-4+WF1+WFE2-3 is sound and ground-complete for BCCS modulo $\simeq_{\mathrm{WF}}$ (see also [10]). Moreover, by Theo. 2 and Theo. 3, we have:

Corollary 1. If $|A|=\infty$, then the axiomatization $A 1-4+W F 1+W F E 2-3$ is $\omega$-complete for $B C C S(A)$ modulo $\simeq_{\mathrm{WF}}$.

Corollary 2. If $|A|<\infty$, then the axiomatization A1-4+WF1+WFE2-3+WFE $E_{A}$ is $\omega$ complete for $B C C S(A)$ modulo $\simeq_{\mathrm{WF}}$. 


\section{Weak Impossible Futures Semantics}

Weak impossible futures semantics is closely related to weak failures semantics. Only, instead of the set of actions in the second argument of a weak failure pair (see Def. 2), an impossible future pair contains a set of traces.

\section{Definition 5 (Weak impossible futures).}

- A sequence $a_{1} \cdots a_{k} \in A^{*}$, with $k \geq 0$, is a trace of a process $p_{0}$ if there is a path $p_{0} \Rightarrow \stackrel{a_{1}}{\rightarrow} \Rightarrow \cdots \Rightarrow \stackrel{a_{k}}{\rightarrow} \Rightarrow p_{k}$. Let $\mathcal{T}(p)$ denote the set of traces of process $p$.

- A pair $\left(a_{1} \cdots a_{k}, B\right)$, with $k \geq 0$ and $B \subseteq A^{*}$, is a weak impossible future of a process $p_{0}$ if there is a path $p_{0} \Rightarrow \stackrel{a_{1}}{\rightarrow} \Rightarrow \cdots \Rightarrow \stackrel{a_{k}}{\rightarrow} \Rightarrow p_{k}$ with $\mathcal{T}\left(p_{k}\right) \cap B=\emptyset$.

- $p \preceq$ WIF $q$ if (1) the weak impossible futures of $p$ are also weak impossible futures of $q$, (2) $\mathcal{T}(p)=\mathcal{T}(q)$ and (3) $p \stackrel{\tau}{\rightarrow}$ implies that $q \stackrel{\tau}{\rightarrow}$.

$-\simeq_{\mathrm{WIF}}=\preceq_{\mathrm{WIF}} \cap \preceq_{\mathrm{WIF}}^{-1}$.

$\preceq_{\mathrm{WIF}}$ is a precongruence, and $\simeq_{\mathrm{WF}}$ a congruence, for BCCS [17].

A sound and ground-complete axiomatization for $\preceq_{\text {WIF }}$ is obtained by replacing axiom WF3 in Tab. 1 by the following axiom (cf. [17], where a slightly more complicated, but equivalent, axiomatization is given):

$$
\text { WIF3 } x \preccurlyeq \tau x
$$

However, surprisingly, there is no finite sound and ground-complete axiomatization for

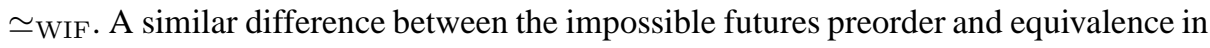
the concrete case (so in the absence of $\tau$ ) was found earlier in [3]. We note that, since weak impossible futures semantics is not coarser than ready simulation semantics, the algorithm from $[1,8]$, to generate an axiomatization for the equivalence from the one for the preorder, does not work in this case.

We have also proved that the sound and ground-complete axiomatization for BCCS modulo $\preceq_{\mathrm{WIF}}$ is $\omega$-complete in case $|A|=\infty$. And that there is no such finite basis for the inequational theory of BCCS modulo $\preceq_{\mathrm{WIF}}$ in case $|A|<\infty$. Again, these results correspond to (in)axiomatizability results for the impossible futures preorder in the concrete case [3].

Theorem 4. There is no finite sound and ground-complete axiomatization for $\mathrm{BCCS}(\mathrm{A})$ modulo $\simeq \mathrm{WIF}$.

Theorem 5. If $|A|=\infty$, then A1-4+WF1-2+WIF3 is $\omega$-complete for BCCS(A) mod$u l o \simeq \mathrm{WIF}$.

Theorem 6. If $|A|<\infty$, then the inequational theory of $\mathrm{BCCS}(\mathrm{A})$ modulo $\preceq_{\mathrm{WIF}}$ does not have a finite basis.

Concluding, in spite of the close resemblance between weak failures and weak impossible futures semantics, there is a striking difference between their axiomatizability properties. 


\section{References}

1. L. Aceto, W. Fokkink and A. Ingólfsdóttir (2007): Ready to preorder: Get your BCCSP axiomatization for free! In Proc. CALCO'07, LNCS 4624, Springer, pp. 65-79.

2. S.D. Brookes, C.A.R. Hoare \& A.W. Roscoe (1984): A theory of communicating sequential processes. Journal of the ACM 31(3), pp. 560-599.

3. T. Chen and W.J. Fokkink (2008): On the axiomatizability of impossible futures: Preorder versus equivalence. Under submission.

4. T. Chen, W.J. Fokkink and R.L. van Glabbeek (2008): On Finite Bases for Weak Semantics: Failures versus Impossible Futures. Full version of current paper. Available at http:// theory.stanford.edu/ rvg/abstracts.html\# 75 .

5. T. Chen, W.J. Fokkink, B. Luttik and S. Nain (2007): On finite alphabets and infinite bases. Information and Computation, CONCUR'06 special issue, To appear.

6. R. De Nicola \& M. Hennessy (1984): Testing equivalences for processes. Theoretical Computer Science 34, pp. 83-133.

7. W. Fokkink and S. Nain (2005): A finite basis for failure semantics. In Proc. ICALP'05, LNCS 3580, Springer, pp. 755-765.

8. D. de Frutos-Escrig and C. Gregorio-Rodríguez (2007): Algebraic and coinductive characterizations of semantics provide general results for free. In Proc. NWPT'07.

9. R.J. van Glabbeek (1993): The linear time - branching time spectrum II. The semantics of sequential systems with silent moves. In Proc. CONCUR'93, LNCS 715, Springer, pp. 66-81.

10. R.J. van Glabbeek (1997): Notes on the methodology of CCS and CSP. Theoretical Computer Science, 177(2), pp. 329-349.

11. R.J. van Glabbeek (2001): The linear time - branching time spectrum I. The semantics of concrete, sequential processes. In Handbook of Process Algebra, Elsevier, pp. 3-99.

12. R.J. van Glabbeek and M. Voorhoeve (2006): Liveness, fairness and impossible futures. In Proc. CONCUR'06, LNCS 4137, Springer, pp. 126-141.

13. J.F. Groote (1990): A new strategy for proving $\omega$-completeness with applications in process algebra. In Proc. CONCUR'90, LNCS 458, Springer, pp. 314-331.

14. A. Rensink and W. Vogler (2007): Fair testing. Information and Computation, 205(2), pp. 125-198.

15. W.C. Rounds \& S.D. Brookes (1981): Possible futures, acceptances, refusals and communicating processes. In Proc. FOCS'81, IEEE, pp. 140-149.

16. W. Vogler (1992): Modular construction and partial order semantics of Petri nets. LNCS 625 , Springer.

17. M. Voorhoeve and S. Mauw (2001): Impossible futures and determinism. Information Processing Letters, 80(1), pp. 51-58. 